\title{
A Single Sweep AGE Algorithm on a Variable Mesh Based on Off-Step Discretization for the Solution of Nonlinear Burgers' Equation
}

\author{
R. K. Mohanty ${ }^{1}$ and Jyoti Talwar $^{2}$ \\ ${ }^{1}$ Department of Applied Mathematics, Faculty of Mathematics \& Computer Science, Akbar Bhawan, \\ Chanakyapuri, New Delhi 110021, India \\ ${ }^{2}$ Department of Mathematics, Faculty of Mathematical Sciences, University of Delhi, Delhi 110007, India
}

Correspondence should be addressed to R. K. Mohanty; rmohanty@maths.du.ac.in

Received 4 October 2013; Accepted 1 December 2013; Published 16 January 2014

Academic Editor: Marta B. Rosales

Copyright (C) 2014 R. K. Mohanty and J. Talwar. This is an open access article distributed under the Creative Commons Attribution License, which permits unrestricted use, distribution, and reproduction in any medium, provided the original work is properly cited.

We discuss a new single sweep alternating group explicit iteration method, along with a third-order numerical method based on offstep discretization on a variable mesh to solve the nonlinear ordinary differential equation $y^{\prime \prime}=f\left(x, y, y^{\prime}\right)$ subject to given natural boundary conditions. Using the proposed method, we have solved Burgers' equation both in singular and nonsingular cases, which is the main attraction of our work. The convergence of the proposed method is discussed in detail. We compared the results of the proposed iteration method with the results of the corresponding double sweep alternating group explicit iteration methods to demonstrate computationally the efficiency of the proposed method.

\section{Introduction}

Consider the general nonlinear ordinary differential equation

$$
y^{\prime \prime}=f\left(x, y, y^{\prime}\right), \quad a<x<b
$$

subject to essential boundary conditions

$$
y(a)=A, \quad y(b)=B
$$

where $-\infty<a<b<\infty, A, B$ are finite constants.

We assume that for $x \in[a, b],-\infty<y, z<\infty$

(i) $f(x, y, z)$ is continuous,

(ii) $\partial f / \partial y$ and $\partial f / \partial z$ exist and are continuous,

(iii) $\partial f / \partial y>0$ and $|\partial f / \partial z|<W$ for some positive constant $W$.

These conditions ensure that the boundary value problem (1) and (2) possesses a unique solution (see Keller [1]).

With the advent of parallel computers, scientists are focusing on developing finite difference methods with the property of parallelism. Working on this, in the early 1980s, Evans $[2,3]$ introduced the Group Explicit methods for large linear system of equations. Further he discussed the Alternating Group Explicit (AGE) method to solve periodic parabolic equations in a coupled manner. Mohanty and Evans applied AGE method along with various high order methods $[4,5]$ for the solution of two-point boundary value problems. Later, Sukon and Evans [6] introduced a Two-parameter Alternating Group Explicit (TAGE) method for the two-point boundary value problem with a lower order accuracy scheme. In 2003 Mohanty et al. [7] discussed the application of TAGE method for nonlinear singular two point boundary value problems using a fourth-order difference scheme. In 1990, Evans introduced the Coupled Alternating Group Explicit method [8] and applied it to periodic parabolic equations. Many scientists are applying these parallel algorithms to solve ordinary and partial differential equations [9-11].

Recently, Mohanty [12] has proposed a high order variable mesh method for nonlinear two-point boundary value problem. Mohanty and Khosla $[13,14]$ also devised a new thirdorder accurate arithmetic average variable mesh method for 
the solution of the boundary value problem (1) and (2), using three grid points, which is applicable to both singular and nonsingular problems. No special technique is required to handle singular problems. They also discussed the application of two-parameter double sweep alternating group explicit methods. Here, we discuss a new single sweep group explicit iteration method along with a third-order accurate variable mesh method based on two extra off-step grid points for the solution of the boundary value problem (1) and (2).

\section{Off-Step Discretization}

Consider the solution interval $[a, b]$ with a nonuniform mesh such that $a=x_{0}<x_{1}<\cdots<x_{N}<x_{N+1}=b$. Let $h_{k}=x_{k}-$ $x_{k-1}, k=1(1) N+1$, be the mesh size and let $\sigma_{k}=h_{k+1} / h_{k}>0$, $k=1(1) N$, be the mesh ratio. Grid points are given by $x_{i}=$ $x_{0}+\sum_{k=1}^{i} h_{k}, i=1(1) N+1$. Let $Y_{k}=y\left(x_{k}\right)$ be the exact solution of $y$ at the grid point $x_{k}$ and approximated by $y_{k}$. Let $x_{k+1 / 2}=x_{k}+\left(\sigma_{k} h_{k} / 2\right)$ and $x_{k-1 / 2}=x_{k}-\left(h_{k} / 2\right)$.

The new third-order method is described as follows.

For $y^{\prime \prime}=f$, we first obtain the off-step discretization

$$
\begin{aligned}
Y_{k+1} & -\left(1+\sigma_{k}\right) Y_{k}+\sigma_{k} Y_{k-1} \\
& =\frac{\sigma_{k} h_{k}^{2}}{3}\left[\sigma_{k} f_{k+1 / 2}+\frac{\left(1+\sigma_{k}\right)}{2} f_{k}+f_{k-1 / 2}\right]+T_{k},
\end{aligned}
$$

where $f_{k+1 / 2}=y^{\prime \prime}\left(x_{k+1 / 2}\right), f_{k-1 / 2}=y^{\prime \prime}\left(x_{k-1 / 2}\right)$, and $T_{k}=$ $O\left(h_{k}^{5}\right)$.

Now, let

$$
\begin{gathered}
\bar{Y}_{k+1 / 2}=\frac{1}{2}\left(Y_{k+1}+Y_{k}\right), \\
\bar{Y}_{k-1 / 2}=\frac{1}{2}\left(Y_{k-1}+Y_{k}\right), \\
\bar{Y}_{k+1 / 2}^{\prime}=\frac{1}{\sigma_{k} h_{k}}\left(Y_{k+1}-Y_{k}\right), \\
\bar{Y}_{k-1 / 2}^{\prime}=\frac{1}{h_{k}}\left(Y_{k}-Y_{k-1}\right), \\
\bar{Y}_{k}^{\prime}=\frac{\left(Y_{k+1}-\left(1-\sigma_{k}^{2}\right) Y_{k}-\sigma_{k}^{2} Y_{k-1}\right)}{\left(h_{k} \sigma_{k}\left(\sigma_{k}+1\right)\right)} \\
\bar{f}_{k+1 / 2}=f\left(x_{k+1 / 2}, \bar{Y}_{k+1 / 2}, \bar{Y}_{k+1 / 2}^{\prime}\right), \\
\bar{f}_{k-1 / 2}=f\left(x_{k-1 / 2}, \bar{Y}_{k-1 / 2}, \bar{Y}_{k-1 / 2}^{\prime}\right), \\
\widehat{Y}_{k=Y_{k}=\left(1-\sigma_{k}+\sigma_{k}^{2}\right)}^{h_{k}^{2}}\left(\bar{f}_{k+1 / 2}+\bar{f}_{k-1 / 2}\right), \\
6\left(1+\sigma_{k}\right) \\
\left(1+\sigma_{k}+\sigma_{k}^{2}\right) h_{k}\left(\bar{f}_{k+1 / 2}-\bar{f}_{k-1 / 2}\right), \\
\left.\widehat{f}_{k}, \widehat{Y}_{k}^{\prime}\right) .
\end{gathered}
$$

Then, at each interior grid point $x_{k}, k=1(1) N$, the proposed differential equation (1) is discretized by

$$
\begin{aligned}
& Y_{k+1}-\left(1+\sigma_{k}\right) Y_{k}+\sigma_{k} Y_{k-1} \\
&= \frac{\sigma_{k} h_{k}^{2}}{3}\left[\sigma_{k} \bar{f}_{k+1 / 2}+\left(\frac{1+\sigma_{k}}{2}\right) \hat{f}_{k}+\bar{f}_{k-1 / 2}\right]+\bar{T}_{k}, \\
& \sigma_{k} \neq 1 .
\end{aligned}
$$

With the help of (3), from (5) it is easy to verify that $\bar{T}_{k}=$ $O\left(h_{k}^{5}\right)$, provided $\sigma_{k} \neq 1$. However, for uniform mesh $\sigma_{k}=1$, the local truncation error associated with (5) becomes $\bar{T}_{k}=$ $O\left(h_{k}^{6}\right)$ (see Chawla and Shivakumar [15]).

Note that the proposed method (5) is applicable to both singular and nonsingular problems. We do not require any special technique to handle singular problems (see Mohanty [13]). Further note that, the boundary values are given by $y_{0}=Y_{0}=A$ and $y_{N+1}=Y_{N+1}=B$. If the differential equation (1) is linear we use the proposed single sweep AGE iterative method and in nonlinear case, we use the NewtonAGE iterative method to obtain the solution.

\section{Application to Singular Problems}

3.1. Linear Singular Problems. We now discuss the application of the proposed numerical method (5) to the linear differential equation with variable coefficients

$$
y^{\prime \prime}=D(x) y^{\prime}+g(x), \quad 0<x<1,
$$

where $g(x)$ represents a forcing function.

For $D(x)=-\alpha / x$ and for $\alpha=1$ and 2 , the equation above represents singular equation in cylindrical and spherical symmetry, respectively.

Let us denote

$$
\begin{gathered}
D_{k}=D\left(x_{k}\right), \quad D_{k \pm 1 / 2}=D\left(x_{k \pm 1 / 2}\right), \\
g_{k}=g\left(x_{k}\right), \quad g_{k \pm 1 / 2}=g\left(x_{k \pm 1 / 2}\right), \quad \sigma_{k}=\frac{h_{k+1}}{h_{k}}, \\
R_{k}=\frac{\left(1+\sigma_{k}+\sigma_{k}^{2}\right)}{12} .
\end{gathered}
$$

Therefore applying the method (5) to the differential equation (6) and neglecting error term, we obtain a linear difference equation of the form

$$
a_{k} y_{k-1}+2 b_{k} y_{k}+c_{k} y_{k+1}=J_{k}, \quad k=1(1) N,
$$

where

$$
\begin{gathered}
a_{k}=-\sigma_{k}\left[1+\frac{h_{k}}{3}\left(1+D_{k} R_{k} h_{k}\right) D_{k-1 / 2}+\frac{\sigma_{k} h_{k}}{6} D_{k}\right] \\
2 b_{k}=\left[1+\sigma_{k}-\frac{h_{k}}{3}\left(\sigma_{k}-D_{k} R_{k} h_{k}\right) D_{k+1 / 2}\right. \\
+\frac{\sigma_{k} h_{k}}{3}\left(1+D_{k} R_{k} h_{k}\right) D_{k-1 / 2} \\
\left.-\frac{h_{k}}{6}\left(1-\sigma_{k}^{2}\right) D_{k}\right]
\end{gathered}
$$




$$
\begin{gathered}
c_{k}=\left[-1+\frac{h_{k}}{3}\left(\sigma_{k}-D_{k} R_{k} h_{k}\right) D_{k+1 / 2}+\frac{h_{k}}{6} D_{k}\right] \\
J_{k}=-\frac{\sigma_{k} h_{k}^{2}}{3}\left[\left(\frac{1+\sigma_{k}}{2}\right) g_{k}+\left(\sigma_{k}-D_{k} R_{k} h_{k}\right) g_{k+1 / 2}\right. \\
\left.+\left(1+D_{k} R_{k} h_{k}\right) g_{k-1 / 2}\right] .
\end{gathered}
$$

The linear difference scheme (8) has a local truncation error of $O\left(h_{k}^{5}\right)$ and is free from the terms $1 / x_{k \pm 1}$ and therefore can very easily be solved for $k=1(1) N$ in the region $x \in$ $(0,1)$.

3.2. Nonlinear Singular Problems. Now we consider the application to nonlinear differential equation (1). Neglecting the error term, we may rewrite the nonlinear difference equation (5) as

$$
\begin{aligned}
y_{k+1} & -\left(1+\sigma_{k}\right) y_{k}+\sigma_{k} y_{k-1} \\
& =\frac{\sigma_{k} h_{k}^{2}}{3}\left[\sigma_{k} \bar{f}_{k+1 / 2}+\left(\frac{1+\sigma_{k}}{2}\right) \widehat{f}_{k}+\bar{f}_{k-1 / 2}\right] .
\end{aligned}
$$

Let

$$
\begin{aligned}
\phi_{k} \equiv & -y_{k+1}+\left(1+\sigma_{k}\right) y_{k}-\sigma_{k} y_{k-1} \\
& +\frac{\sigma_{k} h_{k}^{2}}{3}\left[\sigma_{k} \bar{f}_{k+1 / 2}+\left(\frac{1+\sigma_{k}}{2}\right) \widehat{f}_{k}+\bar{f}_{k-1 / 2}\right] \\
\equiv & \phi_{k}\left(y_{k-1}, y_{k}, y_{k+1}\right), \quad k=1(1) N, \sigma_{k} \neq 1 .
\end{aligned}
$$

We denote

$$
\begin{gathered}
\mathbf{y}=\left[\begin{array}{c}
y_{1} \\
y_{2} \\
\vdots \\
\vdots \\
y_{N}
\end{array}\right]_{N \times 1}, \quad \varphi(\mathbf{y})=\left[\begin{array}{c}
\phi_{1}(\mathbf{y}) \\
\phi_{2}(\mathbf{y}) \\
\vdots \\
\vdots \\
\phi_{N}(\mathbf{y})
\end{array}\right]_{N \times 1}, \\
a_{k}(\mathbf{y})=\frac{\partial \phi_{k}}{\partial y_{k-1}}, \quad k=2(1) N \\
2 b_{k}(\mathbf{y})=\frac{\partial \phi_{k}}{\partial y_{k}}, \quad k=1(1) N \\
c_{k}(\mathbf{y})=\frac{\partial \phi_{k}}{\partial y_{k+1}}, \quad k=1(1) N-1 .
\end{gathered}
$$

The Jacobian $\varphi(\mathbf{y})$ may be represented as

$$
\begin{aligned}
\mathbf{T} & \equiv \frac{\partial \boldsymbol{\varphi}(\mathbf{y})}{\partial y} \\
& =\left[\begin{array}{ccccc}
2 b_{1}(\mathbf{y}) & c_{1}(\mathbf{y}) & & & \mathbf{0} \\
a_{2}(\mathbf{y}) & 2 b_{2}(\mathbf{y}) & c_{2}(\mathbf{y}) & & \\
& \ddots & \ddots & \ddots & \\
& & \ddots & \ddots & c_{N-1}(\mathbf{y}) \\
\mathbf{0} & & & a_{N}(\mathbf{y}) & 2 b_{N}(\mathbf{y})
\end{array}\right]_{N \times N}
\end{aligned}
$$

\section{Single Sweep AGE Method}

4.1. Description of the Method. In this section we discuss the single sweep AGE iteration method. Using the boundary conditions $y_{0}=A, y_{N+1}=B$, the linear difference equation (8) may be written in the matrix form as

$$
\text { Ay }=\text { RH, }
$$

where

$$
\begin{gathered}
\mathbf{A}=\left[\begin{array}{ccccc}
2 b_{1} & c_{1} & & & \mathbf{0} \\
a_{2} & 2 b_{2} & c_{2} & & \\
& & \ddots & & \\
& & a_{N-1} & 2 b_{N-1} & c_{N-1} \\
\mathbf{0} & & a_{N} & 2 b_{N}
\end{array}\right]_{N \times N} \\
\mathbf{y}=\left[\begin{array}{c}
y_{1} \\
y_{2} \\
\vdots \\
\vdots \\
y_{N}
\end{array}\right]_{N \times 1},
\end{gathered}
$$

$$
\mathbf{R H}=\left[\begin{array}{c}
J_{1}-a_{1} y_{0} \\
J_{2} \\
\vdots \\
\vdots \\
J_{N}-c_{N} y_{N+1}
\end{array}\right]_{N \times 1}=\left[\begin{array}{c}
R H_{1} \\
R H_{2} \\
\vdots \\
\vdots \\
R H_{N}
\end{array}\right]_{N \times 1} \text { (say). }
$$

To implement the single sweep AGE iterative method, we split the coefficient matrix $\mathbf{A}$ into two submatrices $\mathbf{A}=\mathbf{G}_{1}+$ $\mathbf{G}_{2}$, where $\mathbf{G}_{1}$ and $\mathbf{G}_{2}$ satisfy the following conditions.

(i) $\mathbf{G}_{1}+\omega_{1} \mathbf{I}$ and $\mathbf{G}_{2}+\omega_{2} \mathbf{I}$ are nonsingular for suitable choice of $\omega_{1}>0$ and $\omega_{2}>0$.

(ii) For any vectors $\nu_{1}$ and $\nu_{2}$ and $\omega_{1}>0, \omega_{2}>0$, it is "convenient" to solve the system explicitly; that is, $\mathbf{z}_{1}=\left(\mathbf{G}_{1}+\omega_{1} \mathbf{I}\right)^{-1} v_{1}$ and $\mathbf{z}_{2}=\left(\mathbf{G}_{2}+\omega_{2} \mathbf{I}\right)^{-1} v_{2}$ for vectors $\mathbf{z}_{1}$ and $\mathbf{z}_{2}$, respectively.

We will be concerned here with the situation where $\mathbf{G}_{1}$ and $\mathbf{G}_{2}$ are small $(2 \times 2)$ block systems.

Now we discuss the case when $N$ is even (with $x_{0}=0$, $x_{N+1}=1$ ).

Let

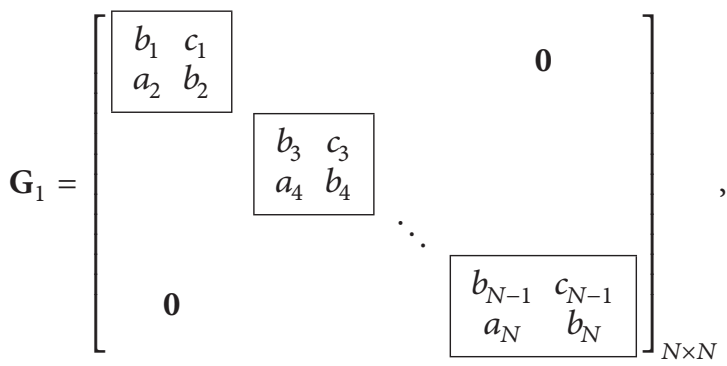




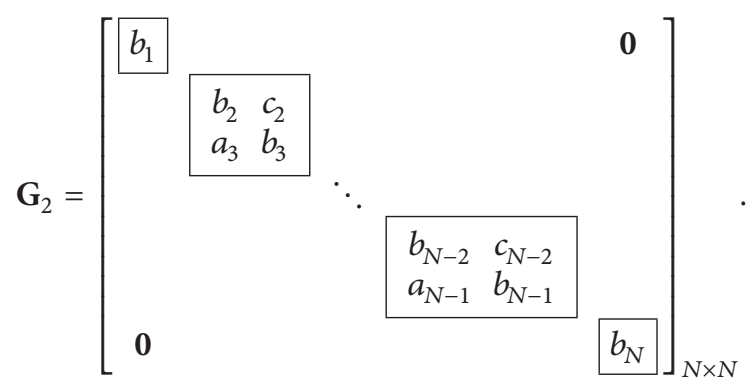

So that the system (14) can be rewritten as

$$
\left(\mathbf{G}_{1}+\mathbf{G}_{2}\right) \mathbf{y}=\mathbf{R H}
$$

Then a two-parameter AGE method for solving the afore mentioned system may be written as

$$
\begin{aligned}
\left(\mathbf{G}_{1}\right. & \left.+\omega_{1} \mathbf{I}\right) \mathbf{z}^{(s)} \\
& =\mathbf{R} \mathbf{H}-\left(\mathbf{G}_{2}-\omega_{1} \mathbf{I}\right) \mathbf{y}^{(s)}, \quad s=0,1,2, \ldots, \\
\left(\mathbf{G}_{2}\right. & \left.+\omega_{2} \mathbf{I}\right) \mathbf{y}^{(s+1)} \\
& =\mathbf{R} \mathbf{H}-\left(\mathbf{G}_{1}-\omega_{2} \mathbf{I}\right) \mathbf{z}^{(s)}, \quad s=0,1,2, \ldots,
\end{aligned}
$$

where $\mathbf{z}^{(s)}$ is an intermediate vector.

Eliminating $\mathbf{z}^{(s)}$ and combining (18) and (19), we obtain the iterative method

$$
\begin{aligned}
\left(\mathbf{G}_{2}\right. & \left.+\omega_{2} \mathbf{I}\right) \mathbf{y}^{(s+1)} \\
= & {\left[\mathbf{I}-\left(\omega_{1}+\omega_{2}\right)\left(\mathbf{G}_{1}+\omega_{1} \mathbf{I}\right)^{-1}\right]\left(\mathbf{G}_{2}-\omega_{1} \mathbf{I}\right) \mathbf{y}^{(s)} } \\
& +\left(\omega_{1}+\omega_{2}\right)\left(\mathbf{G}_{1}+\omega_{1} \mathbf{I}\right)^{-1} \mathbf{R H}, \quad s=0,1,2, \ldots,
\end{aligned}
$$

or

$$
\mathbf{u}^{(s+1)}=\mathbf{T}_{w} \mathbf{y}^{(s)}+\mathbf{R} \mathbf{H}_{w}, \quad s=0,1,2, \ldots,
$$

where

$$
\begin{aligned}
\mathbf{T}_{w}=\left(\mathbf{G}_{2}+\omega_{2} \mathbf{I}\right)^{-1} & \times\left[\left(\mathbf{G}_{2}-\omega_{1} \mathbf{I}\right)\right. \\
& \left.\quad-\left(\omega_{1}+\omega_{2}\right)\left(\mathbf{G}_{1}+\omega_{1} \mathbf{I}\right)^{-1}\left(\mathbf{G}_{2}-\omega_{1} \mathbf{I}\right)\right], \\
& \quad \mathbf{R H}_{w}=\left(\omega_{1}+\omega_{2}\right)\left(\mathbf{G}_{2}+\omega_{2} \mathbf{I}\right)^{-1}\left(\mathbf{G}_{1}+\omega_{1} \mathbf{I}\right)^{-1} \mathbf{R H} .
\end{aligned}
$$

The new iterative method (20) or (21) is called the twoparameter single sweep AGE iterative method and the matrix $\mathbf{T}_{w}$ is called the iteration matrix.

Now we discuss the single sweep AGE algorithm, when $N$ is even.

For simplicity, we denote

$$
\begin{array}{r}
p_{k}=b_{k}+\omega_{1}, \quad q_{k}=b_{k}-\omega_{1}, \quad r_{k}=b_{k}+\omega_{2} \\
\text { for } k=1(1) N, \quad \text { for }\left(p_{k} p_{k+1}-c_{k} a_{k+1}\right) \neq 0,
\end{array}
$$

and we define $d_{k}=1 /\left(p_{k} p_{k+1}-c_{k} a_{k+1}\right)$ for $k=1(1) N-1$.
By carrying out the necessary algebra in (20), we obtain the following algorithm.

For $k=1$,

$$
\begin{aligned}
y_{1}^{(s+1)}=\left(q_{1} y_{1}^{(s)}-\left(\omega_{1}+\omega_{2}\right) d_{1}\right. \\
\quad \times\left[-c_{1} q_{2} y_{2}^{(s)}-c_{1} c_{2} y_{3}^{(s)}+p_{2} q_{1} y_{1}^{(s)}\right. \\
\left.\left.\quad+c_{1} R H_{2}-p_{2} R H_{1}\right]\right) \\
\quad \times\left(r_{1}\right)^{-1}, \quad s=0,1,2, \ldots
\end{aligned}
$$

For $k=2(2) N-2$.

Let

$$
\begin{gathered}
\Delta=r_{k} r_{k+1}-c_{k} a_{k+1} \neq 0, \\
S_{1}=q_{k} y_{k}^{(s)}+c_{k} y_{k+1}^{(s)}-\left(\omega_{1}+\omega_{2}\right) d_{k-1} \\
\times\left[-a_{k-1} a_{k} y_{k-2}^{(s)}-a_{k} q_{k-1} y_{k-1}^{(s)}+p_{k-1} q_{k} y_{k}^{(s)}\right. \\
\left.+p_{k-1} c_{k} y_{k+1}^{(s)}+a_{k} R H_{k-1}-p_{k-1} R H_{k}\right], \\
S_{2}=a_{k+1} y_{k}^{(s)}+q_{k+1} y_{k+1}^{(s)}-\left(\omega_{1}+\omega_{2}\right) d_{k+1} \\
\times\left[p_{k+2} a_{k+1} y_{k}^{(s)}+p_{k+2} q_{k+1} y_{k+1}^{(s)}\right. \\
-c_{k+1} q_{k+2} y_{k+2}^{(s)}-c_{k+1} c_{k+2} y_{k+3}^{(s)} \\
\left.-p_{k+2} R H_{k+1}+c_{k+1} R H_{k+2}\right]
\end{gathered}
$$

and then

$$
\begin{aligned}
& y_{k}^{(s+1)}=\frac{\left(S_{1} r_{k+1}-S_{2} c_{k}\right)}{\Delta}, \quad s=0,1,2, \ldots \\
& y_{k+1}^{(s+1)}=\frac{\left(S_{2} r_{k}-S_{1} a_{k+1}\right)}{\Delta}, \quad s=0,1,2, \ldots
\end{aligned}
$$

Finally, for $k=N$,

$$
\begin{aligned}
y_{N}^{(s+1)}=( & q_{N} y_{N}^{(s)}-\left(\omega_{1}+\omega_{2}\right) d_{N-1} \\
& \times\left[-a_{N-1} a_{N} y_{N-2}^{(s)}-a_{N} q_{N-1} y_{N-1}^{(s)}+p_{N-1} q_{N} y_{N}^{(s)}\right. \\
& \left.\left.\quad+a_{N} R H_{N-1}-p_{N-1} R H_{N}\right]\right) \\
& \times\left(r_{N}\right)^{-1}, \quad s=0,1,2, \ldots
\end{aligned}
$$

Similarly, we can write the single sweep AGE algorithm when $N$ is odd.

4.2. Convergence Analysis. The single sweep AGE iteration method is given by

$$
\begin{aligned}
\left(\mathbf{G}_{2}\right. & \left.+\omega_{2} \mathbf{I}\right) \mathbf{y}^{(s+1)} \\
= & {\left[\mathbf{I}-\left(\omega_{1}+\omega_{2}\right)\left(\mathbf{G}_{1}+\omega_{1} \mathbf{I}\right)^{-1}\right]\left(\mathbf{G}_{2}-\omega_{1} \mathbf{I}\right) \mathbf{y}^{(s)} } \\
& +\left(\omega_{1}+\omega_{2}\right)\left(\mathbf{G}_{1}+\omega_{1} \mathbf{I}\right)^{-1} \mathbf{R} \mathbf{H}, \quad s=0,1,2, \ldots,
\end{aligned}
$$


or

$$
\mathbf{y}^{(s+1)}=\mathbf{T}_{w} \mathbf{y}^{(s)}+\mathbf{R} \mathbf{H}_{w}, \quad s=0,1,2, \ldots,
$$

where

$$
\begin{aligned}
\mathbf{T}_{w}= & \left(\mathbf{G}_{2}+\omega_{2} \mathbf{I}\right)^{-1} \\
\times & {\left[\left(\mathbf{G}_{2}-\omega_{1} \mathbf{I}\right)\right.} \\
& \left.\quad-\left(\omega_{1}+\omega_{2}\right)\left(\mathbf{G}_{1}+\omega_{1} \mathbf{I}\right)^{-1}\left(\mathbf{G}_{2}-\omega_{1} \mathbf{I}\right)\right], \\
& \quad \mathbf{R H}_{w}=\left(\omega_{1}+\omega_{2}\right)\left(\mathbf{G}_{2}+\omega_{2} \mathbf{I}\right)^{-1}\left(\mathbf{G}_{1}+\omega_{1} \mathbf{I}\right)^{-1} \mathbf{R H} .
\end{aligned}
$$

The matrix $\mathbf{T}_{w}$ is called the iteration matrix.

To prove the convergence of the method, we need to prove that $S\left(\mathbf{T}_{w}\right) \leq 1$, where $S\left(\mathbf{T}_{w}\right)$ denotes the spectral radius of $\mathbf{T}_{w}$.

Lemma 1. Let $h_{k}$ be sufficiently small. Then, the eigenvalues of $\mathbf{G}_{1}$ and $\mathbf{G}_{2}$ are all real.

Proof. Consider

$$
\begin{aligned}
a_{k} & =-\sigma_{k}\left[1+\frac{h_{k}}{3}\left(1+D_{k} R_{k} h_{k}\right) D_{k-1 / 2}+\frac{\sigma_{k} h_{k}}{6} D_{k}\right] \\
& =-\sigma_{k}-O\left(h_{k}\right)<0, \quad \text { for sufficiently small } h_{k}, \\
c_{k} & =\left[-1+\frac{h_{k}}{3}\left(\sigma_{k}-D_{k} R_{k} h_{k}\right) D_{k+1 / 2}+\frac{h_{k}}{6} D_{k}\right] \\
& =-1+O\left(h_{k}\right)<0, \quad \text { for sufficiently small } h_{k} .
\end{aligned}
$$

Therefore, we have $a_{k+1} c_{k}>0$, for $k=1(2) N-1$.

Let $\lambda_{i}, i=1(1) N$, be the eigenvalues of $\mathbf{G}_{1}$. Then $\lambda_{i}$ 's are the roots of the quadratic equation

$$
\lambda_{i}^{2}-\left(b_{i}+b_{i+1}\right) \lambda_{i}+\left(b_{i} b_{i+1}-a_{i+1} c_{i}\right)=0 .
$$

Simplifying, we get

$$
\lambda_{i}=\frac{1}{2}\left[\left(b_{i}+b_{i+1}\right) \pm \sqrt{\left(b_{i}-b_{i+1}\right)^{2}+4 a_{i+1} c_{i}}\right] .
$$

The discriminants of the quadratic equations are

$$
\left(b_{i}-b_{i+1}\right)^{2}+4 a_{i+1} c_{i}>0, \quad k=1(2) N-1 .
$$

Hence, the eigenvalues of $\mathbf{G}_{1}$ are real.

In a similar manner, we can show that the eigenvalues of $\mathbf{G}_{2}$ are real.

Now we give the sufficient condition for the convergence of the method.

Theorem 2. Let $\lambda_{i}$ and $\mu_{i}, i=1(1) N$, be the eigenvalues of $\mathbf{G}_{1}$ and $\mathbf{G}_{2}$, respectively. If

$$
\begin{gathered}
\omega_{1}>\max \left\{0,-\lambda_{1}, \ldots,-\lambda_{N}\right\}, \\
\omega_{2}>\max \left\{0,-\mu_{1}, \ldots,-\mu_{N}\right\}, \\
\omega_{2}-2 \min _{i} \lambda_{i}<\omega_{1}<\omega_{2}+2 \min _{i} \mu_{i},
\end{gathered}
$$

then the iterative method is convergent for the system (14).
Proof. Let

$$
\begin{aligned}
\mathbf{D} & =\operatorname{diag}\left(1, \frac{c_{1}}{a_{2}}, \frac{c_{1} c_{2}}{a_{2} a_{3}}, \ldots, \frac{c_{1} c_{2} \cdots c_{N-1}}{a_{2} a_{3} \cdots a_{N}}\right) \\
& \equiv \operatorname{diag}\left(d_{1}, d_{2}, d_{3}, \ldots, d_{N}\right) .
\end{aligned}
$$

Since the off-diagonal entries of $\mathbf{A}$ are negative, therefore $a_{k+1} c_{k}>0, k=1, \ldots, N-1$. Therefore, the diagonal entries of $\mathbf{D}$ are positive.

The iteration matrix is given by

$$
\begin{aligned}
\mathbf{T}_{w}= & \left(\mathbf{G}_{2}+\omega_{2} \mathbf{I}\right)^{-1} \\
& \times\left[\left(\mathbf{G}_{2}-\omega_{1} \mathbf{I}\right)\right. \\
& \left.\quad-\left(\omega_{1}+\omega_{2}\right)\left(\mathbf{G}_{1}+\omega_{1} \mathbf{I}\right)^{-1}\left(\mathbf{G}_{2}-\omega_{1} \mathbf{I}\right)\right] \\
= & \left(\mathbf{G}_{2}+\omega_{2} \mathbf{I}\right)^{-1} \\
& \times\left[\mathbf{I}-\left(\omega_{1}+\omega_{2}\right)\left(\mathbf{G}_{1}+\omega_{1} \mathbf{I}\right)^{-1}\right]\left(\mathbf{G}_{2}-\omega_{1} \mathbf{I}\right) .
\end{aligned}
$$

Define

$$
\begin{aligned}
\mathbf{T}_{w}^{*}= & \left(\mathbf{G}_{2}+\omega_{2} \mathbf{I}\right) \mathbf{T}_{w}\left(\mathbf{G}_{2}+\omega_{2} \mathbf{I}\right)^{-1} \\
= & {\left[\mathbf{I}-\left(\omega_{1}+\omega_{2}\right)\left(\mathbf{G}_{1}+\omega_{1} \mathbf{I}\right)^{-1}\right] } \\
\times & \left(\mathbf{G}_{2}-\omega_{1} \mathbf{I}\right)\left(\mathbf{G}_{2}+\omega_{2} \mathbf{I}\right)^{-1} \\
\mathbf{S}\left(\mathbf{T}_{w}\right)= & \mathbf{S}\left(\mathbf{T}_{w}^{*}\right)=\mathbf{S}\left(\mathbf{D}^{1 / 2} \mathbf{T}_{w}^{*} \mathbf{D}^{-1 / 2}\right) \\
\mathbf{D}^{1 / 2} \mathbf{T}_{w}^{*} \mathbf{D}^{-1 / 2}= & {\left[\mathbf{I}-\left(\omega_{1}+\omega_{2}\right)\left(\overline{\mathbf{G}}_{1}+\omega_{1} \mathbf{I}\right)^{-1}\right] } \\
& \times\left(\overline{\mathbf{G}}_{2}-\omega_{1} \mathbf{I}\right)\left(\overline{\mathbf{G}}_{2}+\omega_{2} \mathbf{I}\right)^{-1},
\end{aligned}
$$

It is easy to verify that $\overline{\mathbf{G}}_{1}$ and $\overline{\mathbf{G}}_{2}$ are symmetric. Therefore, the matrices $\left(\overline{\mathbf{G}}_{2}-\omega_{1} \mathbf{I}\right)\left(\overline{\mathbf{G}}_{2}+\omega_{2} \mathbf{I}\right)^{-1}$ and $\left[\mathbf{I}-\left(\omega_{1}+\right.\right.$ $\left.\left.\omega_{2}\right)\left(\overline{\mathbf{G}}_{1}+\omega_{1} \mathbf{I}\right)^{-1}\right]$ are also symmetric. Hence,

$$
\begin{gathered}
\left\|\left(\overline{\mathbf{G}}_{2}-\omega_{1} \mathbf{I}\right)\left(\overline{\mathbf{G}}_{2}+\omega_{2} \mathbf{I}\right)^{-1}\right\|_{2}=\max _{\mu_{i} \in \sigma\left(\overline{\mathbf{G}}_{2}\right)}\left|\frac{\left(\mu_{i}-\omega_{1}\right)}{\left(\mu_{i}+\omega_{2}\right)}\right| \\
\left\|\left[\mathbf{I}-\left(\omega_{1}+\omega_{2}\right)\left(\overline{\mathbf{G}}_{1}+\omega_{1} \mathbf{I}\right)^{-1}\right]\right\|_{2} \\
=\|\left[\left(\overline{\mathbf{G}}_{1}+\omega_{1} \mathbf{I}\right)\left(\overline{\mathbf{G}}_{1}+\omega_{1} \mathbf{I}\right)^{-1}\right. \\
\left.\quad-\left(\omega_{1}+\omega_{2}\right)\left(\overline{\mathbf{G}}_{1}+\omega_{1} \mathbf{I}\right)^{-1}\right] \|_{2} \\
=\left\|\left[\left(\overline{\mathbf{G}}_{1}-\omega_{2} \mathbf{I}\right)\right]\left[\left(\overline{\mathbf{G}}_{1}+\omega_{1} \mathbf{I}\right)^{-1}\right]\right\|_{2} .
\end{gathered}
$$


TABle 1: Example 1.

\begin{tabular}{|c|c|c|c|c|c|c|c|c|}
\hline \multirow{2}{*}{$N$} & \multicolumn{3}{|c|}{ TAGE } & \multicolumn{3}{|c|}{ Single sweep AGE } & \multirow{2}{*}{ MAE } & \multirow{2}{*}{ RMS errors } \\
\hline & $\omega_{1}$ & $\omega_{2}$ & Iter & $\omega_{1}$ & $\omega_{2}$ & Iter & & \\
\hline \multicolumn{9}{|c|}{$\alpha=1, \sigma=0.7$} \\
\hline 10 & 0.441 & 0.44 & 38 & 0.47 & 0.34 & 20 & $0.4062(-03)$ & $0.2435(-03)$ \\
\hline 20 & 0.286 & 0.286 & 59 & 0.33 & 0.25 & 33 & $0.3604(-03)$ & $0.1558(-03)$ \\
\hline 30 & 0.25 & 0.247 & 77 & 0.32 & 0.23 & 44 & $0.3592(-03)$ & $0.1269(-03)$ \\
\hline 40 & 0.218 & 0.218 & 75 & 0.24 & 0.20 & 47 & $0.3592(-03)$ & $0.1099(-03)$ \\
\hline 50 & 0.203 & 0.203 & 86 & 0.26 & 0.21 & 54 & $0.3592(-03)$ & $0.9829(-04)$ \\
\hline 60 & 0.202 & 0.199 & 96 & 0.26 & 0.20 & 62 & $0.3592(-03)$ & $0.8973(-04)$ \\
\hline 70 & 0.194 & 0.194 & 100 & 0.26 & 0.20 & 65 & $0.3592(-03)$ & $0.8307(-04)$ \\
\hline 80 & 0.149 & 0.149 & 79 & 0.25 & 0.17 & 62 & $0.3592(-03)$ & $0.7771(-04)$ \\
\hline \multicolumn{9}{|c|}{$\alpha=2, \sigma=1.1$} \\
\hline 10 & 0.529 & 0.524 & 39 & 0.51 & 0.40 & 23 & $0.2990(-02)$ & $0.2910(-02)$ \\
\hline 20 & 0.298 & 0.297 & 78 & 0.34 & 0.26 & 44 & $0.9774(-03)$ & $0.9580(-03)$ \\
\hline 30 & 0.213 & 0.213 & 113 & 0.29 & 0.20 & 63 & $0.6774(-03)$ & $0.6672(-03)$ \\
\hline 40 & 0.169 & 0.168 & 146 & 0.16 & 0.15 & 81 & $0.5925(-03)$ & $0.5855(-03)$ \\
\hline 50 & 0.142 & 0.141 & 178 & 0.14 & 0.13 & 101 & $0.5633(-03)$ & $0.5579(-03)$ \\
\hline 60 & 0.124 & 0.124 & 180 & 0.14 & 0.12 & 113 & $0.5525(-03)$ & $0.5481(-03)$ \\
\hline 70 & 0.112 & 0.112 & 201 & 0.14 & 0.12 & 118 & $0.5484(-03)$ & $0.5446(-03)$ \\
\hline 80 & 0.103 & 0.103 & 221 & 0.13 & 0.10 & 128 & $0.5469(-03)$ & $0.5435(-03)$ \\
\hline \multicolumn{9}{|c|}{$\alpha=1, \sigma=1.0$} \\
\hline 10 & 0.57 & 0.37 & 47 & 0.46 & 0.34 & 26 & $0.4258(-03)$ & $0.3739(-03)$ \\
\hline 20 & 0.31 & 0.23 & 115 & 0.25 & 0.239 & 75 & $0.3371(-04)$ & $0.2934(-04)$ \\
\hline 30 & 0.20 & 0.19 & 204 & 0.203 & 0.183 & 128 & $0.7197(-05)$ & $0.6256(-05)$ \\
\hline 40 & 0.17 & 0.15 & 309 & 0.19 & 0.17 & 225 & $0.2367(-05)$ & $0.2058(-05)$ \\
\hline 50 & 0.14 & 0.14 & 445 & 0.1749 & 0.173 & 362 & $0.9928(-06)$ & $0.8634(-06)$ \\
\hline 60 & 0.13 & 0.13 & 623 & 0.179 & 0.164 & 503 & $0.4864(-06)$ & $0.4232(-06)$ \\
\hline 70 & 0.125 & 0.124 & 842 & 0.177 & 0.163 & 681 & $0.2654(-06)$ & $0.2310(-06)$ \\
\hline 80 & 0.124 & 0.123 & 1117 & 0.176 & 0.163 & 888 & $0.1567(-06)$ & $0.1365(-06)$ \\
\hline \multicolumn{9}{|c|}{$\alpha=2, \sigma=1.0$} \\
\hline 10 & 0.59 & 0.42 & 40 & 0.45 & 0.39 & 24 & $0.5725(-03)$ & $0.5344(-03)$ \\
\hline 20 & 0.32 & 0.25 & 86 & 0.34 & 0.25 & 45 & $0.4488(-04)$ & $0.4152(-04)$ \\
\hline 30 & 0.20 & 0.19 & 131 & 0.21 & 0.18 & 73 & $0.9532(-05)$ & $0.8792(-05)$ \\
\hline 40 & 0.16 & 0.14 & 180 & 0.16 & 0.14 & 99 & $0.3125(-05)$ & $0.2877(-05)$ \\
\hline 50 & 0.12 & 0.12 & 228 & 0.11 & 0.10 & 126 & $0.1307(-05)$ & $0.1202(-05)$ \\
\hline 60 & 0.1 & 0.1 & 274 & 0.10 & 0.09 & 151 & $0.6392(-06)$ & $0.5876(-06)$ \\
\hline 70 & 0.08 & 0.08 & 332 & 0.09 & 0.08 & 176 & $0.3485(-06)$ & $0.1890(-06)$ \\
\hline 80 & 0.07 & 0.07 & 382 & 0.08 & 0.07 & 202 & $0.2058(-06)$ & $0.1890(-06)$ \\
\hline
\end{tabular}

Also, $\left(\overline{\mathbf{G}}_{1}-\omega_{2} \mathbf{I}\right)\left(\overline{\mathbf{G}}_{1}+\omega_{1} \mathbf{I}\right)^{-1}$ is symmetric, and therefore

$$
\left\|\left[\left(\overline{\mathbf{G}}_{1}-\omega_{2} \mathbf{I}\right)\right]\left[\left(\overline{\mathbf{G}}_{1}+\omega_{1} \mathbf{I}\right)^{-1}\right]\right\|_{2}=\max _{\lambda_{i} \in \sigma\left(\overline{\mathbf{G}}_{1}\right)}\left|\frac{\left(\lambda_{i}-\omega_{2}\right)}{\left(\lambda_{i}+\omega_{1}\right)}\right| .
$$

Therefore, we have

$$
\mathbf{S}\left(\mathbf{T}_{w}\right) \leq \max _{\lambda_{i} \in \sigma\left(\overline{\mathbf{G}}_{1}\right)}\left|\frac{\left(\lambda_{i}-\omega_{2}\right)}{\left(\lambda_{i}+\omega_{1}\right)}\right| \max _{\mu_{i} \in \sigma\left(\overline{\mathbf{G}}_{2}\right)}\left|\frac{\left(\mu_{i}-\omega_{1}\right)}{\left(\mu_{i}+\omega_{2}\right)}\right| .
$$

From (35) and (36), we have $\omega_{1}, \omega_{2}>0$ and $\lambda_{i}+\omega_{1}>0$ for $k=1, \ldots, N$.
Hence,

$$
\frac{\lambda_{i}-\omega_{2}}{\lambda_{i}+\omega_{1}}<1, \quad k=1(1) N .
$$

Also, from (37), we have

$$
\omega_{2}<\omega_{1}+2 \min _{i} \lambda_{i}<\omega_{1}+2 \lambda_{i}, \quad i=1(1) N
$$$$
-1<\frac{\lambda_{i}-\omega_{2}}{\lambda_{i}+\omega_{1}}, \quad k=1(1) N .
$$

Hence, we conclude that

$$
\left|\frac{\lambda_{i}-\omega_{2}}{\lambda_{i}+\omega_{1}}\right|<1, \quad k=1(1) N .
$$


TABLe 2: Example 2.

\begin{tabular}{|c|c|c|c|c|c|c|c|c|}
\hline \multirow{2}{*}{$N$} & \multicolumn{3}{|c|}{ TAGE } & \multicolumn{3}{|c|}{ Single sweep AGE } & \multirow{2}{*}{ MAE } & \multirow{2}{*}{ RMS errors } \\
\hline & $\omega_{1}$ & $\omega_{2}$ & Iter & $\omega_{1}$ & $\omega_{2}$ & Iter & & \\
\hline \multicolumn{9}{|c|}{$R_{e}=10, \sigma=1.4$} \\
\hline 10 & 0.0578 & 0.0585 & 6 & 0.0568 & 0.0568 & 6 & $0.1067(-03)$ & $0.6084(-04)$ \\
\hline 20 & 0.0368 & 0.0374 & 7 & 0.0281 & 0.0285 & 7 & $0.8511(-04)$ & $0.3726(-04)$ \\
\hline 30 & 0.0318 & 0.0321 & 8 & 0.0320 & 0.0315 & 7 & $0.8442(-04)$ & $0.3028(-04)$ \\
\hline 40 & 0.0282 & 0.0286 & 9 & 0.0170 & 0.0170 & 8 & $0.8440(-04)$ & $0.2622(-04)$ \\
\hline 50 & 0.0236 & 0.0239 & 10 & 0.0160 & 0.0166 & 8 & $0.8440(-04)$ & $0.2345(-04)$ \\
\hline 60 & 0.0161 & 0.0162 & 10 & 0.0161 & 0.0166 & 8 & $0.8440(-04)$ & $0.2141(-04)$ \\
\hline \multicolumn{9}{|c|}{$R_{e}=50, \sigma=1.1$} \\
\hline 10 & 0.0152 & 0.0155 & 5 & 0.016 & 0.016 & 4 & $0.8156(-03)$ & $0.3181(-03)$ \\
\hline 20 & 0.0093 & 0.0096 & 6 & 0.009 & 0.0089 & 5 & $0.3228(-04)$ & $0.1508(-04)$ \\
\hline 30 & 0.0063 & 0.0068 & 7 & 0.006 & 0.0059 & 7 & $0.8624(-05)$ & $0.4435(-05)$ \\
\hline 40 & 0.0044 & 0.0045 & 10 & 0.0032 & 0.0042 & 9 & $0.5159(-05)$ & $0.2771(-05)$ \\
\hline 50 & 0.0036 & 0.0035 & 12 & 0.002 & 0.0031 & 12 & $0.4238(-05)$ & $0.2232(-05)$ \\
\hline 60 & 0.0029 & 0.0029 & 10 & 0.0027 & 0.00278 & 15 & $0.3917(-05)$ & $0.1968(-05)$ \\
\hline \multicolumn{9}{|c|}{$R_{e}=50, \sigma=1.0$} \\
\hline 10 & 0.006 & 0.014 & 5 & 0.005 & 0.014 & 4 & $0.3335(-02)$ & $0.1197(-02)$ \\
\hline 20 & 0.012 & 0.012 & 5 & 0.008 & 0.0089 & 4 & $0.3326(-03)$ & $0.9523(-04)$ \\
\hline 30 & 0.008 & 0.008 & 6 & 0.008 & 0.008 & 4 & $0.7487(-04)$ & $0.2147(-04)$ \\
\hline 40 & 0.0061 & 0.0061 & 7 & 0.0051 & 0.0053 & 5 & $0.2502(-04)$ & $0.7150(-05)$ \\
\hline 50 & 0.0049 & 0.0049 & 8 & 0.0058 & 0.0049 & 5 & $0.1055(-04)$ & $0.3012(-05)$ \\
\hline 60 & 0.0041 & 0.0041 & 9 & 0.0043 & 0.0041 & 5 & $0.5186(-05)$ & $0.1478(-05)$ \\
\hline
\end{tabular}

Thus,

$$
\max _{\lambda_{i} \in \sigma\left(\overline{\mathbf{G}}_{1}\right)}\left|\frac{\left(\lambda_{i}-\omega_{2}\right)}{\left(\lambda_{i}+\omega_{1}\right)}\right|<1 .
$$

Similarly, we can prove that

$$
\max _{\mu_{i} \in \sigma\left(\overline{\mathbf{G}}_{2}\right)}\left|\frac{\left(\mu_{i}-\omega_{1}\right)}{\left(\mu_{i}+\omega_{2}\right)}\right|<1 .
$$

Using (44), (48), and (49), we get $\mathbf{S}\left(\mathbf{T}_{w}\right)<1$.

Hence, the convergence of the method (20) follows.

4.3. Application of Single Sweep Newton-AGE Method. Now we discuss the single sweep Newton-AGE iterative method for the nonlinear difference equation (11). We follow the approaches given by Evans $[8,16]$.

Let us define

$$
\begin{gathered}
\mathbf{y}=\left[\begin{array}{c}
y_{1} \\
y_{2} \\
\vdots \\
y_{N}
\end{array}\right]_{N \times 1}, \quad \boldsymbol{\varphi}(\mathbf{y})=\left[\begin{array}{c}
\phi_{1}(\mathbf{y}) \\
\phi_{2}(\mathbf{y}) \\
\vdots \\
\phi_{N}(\mathbf{y})
\end{array}\right]_{N \times 1}, \\
a_{k}(\mathbf{y})=\frac{\partial \phi_{k}}{\partial y_{k-1}}, \quad k=2(1) N
\end{gathered}
$$

$$
\begin{gathered}
2 b_{k}(\mathbf{y})=\frac{\partial \phi_{k}}{\partial y_{k}}, \quad k=1(1) N \\
c_{k}(\mathbf{y})=\frac{\partial \phi_{k}}{\partial y_{k+1}}, \quad k=1(1) N-1 .
\end{gathered}
$$

Then the Jacobian of $\varphi(\mathbf{y})$ can be written as the $N$ th-order tridiagonal matrix

$$
\begin{aligned}
\mathbf{T} & =\frac{\partial \varphi(\mathbf{y})}{\partial \mathbf{y}} \\
& =\left[\begin{array}{ccccc}
2 b_{1}(\mathbf{y}) & c_{1}(\mathbf{y}) & & & \mathbf{0} \\
a_{2}(\mathbf{y}) & 2 b_{2}(\mathbf{y}) & c_{2}(\mathbf{y}) & & \\
& & \ddots & \ddots & \\
\mathbf{0} & & & a_{N}(\mathbf{y}) & 2 b_{N}(\mathbf{y})
\end{array}\right]_{N \times N}
\end{aligned}
$$

Now with any initial vector $\mathbf{y}^{(0)}$, we define

$$
\mathbf{y}^{(s+1)}=\mathbf{y}^{(s)}+\Delta \mathbf{y}^{(s)}, \quad s=0,1,2, \ldots,
$$

where $\Delta \mathbf{y}^{(s)}$ is the solution of the nonlinear system

$$
\mathbf{T} \Delta \mathbf{y}^{(s)}=-\boldsymbol{\varphi}\left(\mathbf{y}^{(s)}\right), \quad s=0,1,2, \ldots
$$


For the single sweep Newton-AGE method, we consider the case when $N$ is even. We split the matrix $\mathbf{T}$ as $\mathbf{T}=\mathbf{T}_{1}+\mathbf{T}_{2}$, where
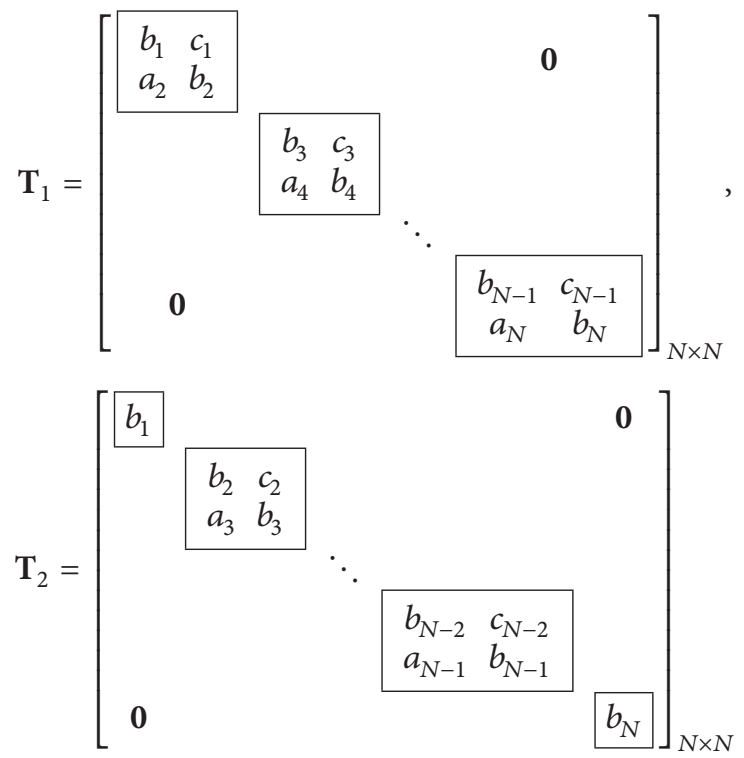

and then we write single sweep Newton-AGE method as

$$
\begin{aligned}
& \left(\mathbf{T}_{2}+\omega_{2} \mathbf{I}\right) \Delta \mathbf{y}^{(s+1)} \\
& =\left[\mathbf{I}-\left(\omega_{1}+\omega_{2}\right)\left(\mathbf{T}_{1}+\omega_{1} \mathbf{I}\right)^{-1}\right]\left(\mathbf{T}_{2}-\omega_{1} \mathbf{I}\right) \Delta \mathbf{y}^{(s)} \\
& \quad-\left(\omega_{1}+\omega_{2}\right)\left(\mathbf{T}_{1}+\omega_{1} \mathbf{I}\right)^{-1} \boldsymbol{\varphi}\left(\mathbf{y}^{(s)}\right), \\
& \quad s=0,1,2, \ldots,
\end{aligned}
$$

where $\omega_{1}>0, \omega_{2}>0$ are relaxation parameters and $\left(\mathbf{T}_{1}+\omega_{1} \mathbf{I}\right)$ and $\left(\mathbf{T}_{2}+\omega_{2} \mathbf{I}\right)$ are nonsingular.

Since $\left(\mathbf{T}_{1}+\omega_{1} \mathbf{I}\right)$ and $\left(\mathbf{T}_{2}+\omega_{2} \mathbf{I}\right)$ consist of $(2 \times 2)$ submatrices, they can be easily inverted as

$$
\begin{aligned}
& \left(\mathbf{T}_{1}+\omega_{1} \mathbf{I}\right)^{-1} \\
& =\left[\begin{array}{cccc}
\frac{\left(\begin{array}{cc}
p_{2} & -c_{1} \\
-a_{2} & p_{1}
\end{array}\right)}{\Delta_{1}} & & \\
& \ddots & & \\
0 & & & \frac{\left(\begin{array}{c}
p_{N}-c_{N-1} \\
-a_{N} p_{N-1}
\end{array}\right)}{\Delta_{N-1}}
\end{array}\right]_{N \times N}, \\
& \left(\mathbf{T}_{2}+\omega_{2} \mathbf{I}\right)^{-1} \\
& =\left[\begin{array}{ccc}
\frac{1}{r_{1}} & & \\
& \frac{\left(\begin{array}{cc}
r_{3} & -c_{2} \\
-a_{3} & r_{2}
\end{array}\right)}{\Delta_{2}} & \\
& 0 & \frac{1}{r_{N}}
\end{array}\right]_{N \times N}
\end{aligned}
$$

with $p_{k}=b_{k}+\omega_{1}, k=1(1) N, \Delta_{k}=p_{k} p_{k+1}-c_{k} a_{k+1}, k=$ $1(2) N-1, r_{k}=b_{k}+\omega_{2}, k=1(1) N$, and $\Delta_{k}=r_{k} r_{k+1}-$ $c_{k} a_{k+1}, \quad k=2(2) N-2$.

The matrices $\left(\mathbf{T}_{2}+\omega_{2} \mathbf{I}\right)^{-1}\left(\mathbf{T}_{1}+\omega_{1} \mathbf{I}\right)^{-1}\left(\mathbf{T}_{2}-\omega_{1} \mathbf{I}\right)$ and $\left(\mathbf{T}_{2}+\right.$ $\left.\omega_{2} \mathbf{I}\right)^{-1}\left(\mathbf{T}_{1}+\omega_{1} \mathbf{I}\right)^{-1}$ can be evaluated in a manner suitable for parallel computing. In order for this method to converge, it is sufficient that the initial vector $\mathbf{y}^{(0)}$ be close to the solution.

Similarly, we can write the single sweep Newton-AGE algorithm when $N$ is odd.

\section{Results and Observations}

We have applied the methods to the following three examples, whose exact solutions are known to us, and have compared the results with the corresponding double sweep AGE and Newton-AGE method [14]. For single sweep Newton-AGE method, we use the technique given by Evans [16]. The right hand side function and boundary conditions may be obtained using the exact solutions. Here, we have taken $\sigma_{k}=\sigma=a$ constant, $k=1(1) N+1$. The value of the first mesh spacing on the left is given by

$$
h_{1}=\frac{(b-a)(1-\sigma)}{\left(1-\sigma^{N+1}\right)}, \quad \sigma \neq 1 .
$$

Therefore, given the value of $N$ and $\sigma$, we can calculate $h_{1}$ from the above relation and the remaining mesh points are determined by $h_{k+1}=\sigma h_{k}, k=1(1) N$. The initial vector $\mathbf{y}^{(0)}=\mathbf{0}$ is used in all iterative methods and the iterations were stopped when the absolute error tolerance $\left|y^{(s+1)}-y^{(s)}\right| \leq$ $10^{-10}$ was achieved.

Example 1 (linear singular problem). Consider

$$
y^{\prime \prime}+\frac{\alpha}{x} y^{\prime}=f(x), \quad 0<x<1
$$

The exact solution is $y(x)=e^{x^{4}}$. The root mean square errors (RMSE), the maximum absolute errors (MAE), and number of iterations both for single and double sweep AGE methods are tabulated in Table 1 for $\alpha=1, \sigma=0.7, \alpha=2, \sigma=1.1$, $\alpha=1, \sigma=1.0$, and $\alpha=2, \sigma=1.0$. The graph of Example 1 of exact solution versus numerical solution is plotted in Figure 1.

Example 2 (Burgers' equation). Consider

$$
v y^{\prime \prime}=\left(y-\frac{1}{2}\right) y^{\prime}, \quad 0<x<1 .
$$

The exact solution is given by $y(x)=(1 / 2)[1-\tanh (x / 4 v)]$, where $v=R_{e}^{-1}$. The RMSE, MAE, and the number of iterations for both single and double sweep AGE methods are tabulated in Table 2 for $R_{e}=50, \sigma=1.1, R_{e}=10, \sigma=1.4$, and $R_{e}=50, \sigma=1.0$. The graph of Example 2 of exact solution versus numerical solution for $N=60, R_{e}=50, \sigma=1.0$ is plotted in Figure 2.

Example 3 (Burgers' equation in polar coordinates). Consider

$$
\frac{1}{R_{e}}\left(y^{\prime \prime}+\frac{\alpha}{x} y^{\prime}-\frac{\alpha}{x^{2}} y\right)=y y^{\prime}+f(x), \quad 0<x<1 .
$$


TABle 3: Example 3.

\begin{tabular}{|c|c|c|c|c|c|c|c|c|}
\hline \multirow{2}{*}{$N$} & \multicolumn{3}{|c|}{ TAGE } & \multicolumn{3}{|c|}{ Single sweep AGE } & \multirow{2}{*}{ MAE } & \multirow{2}{*}{ RMS errors } \\
\hline & $\omega_{1}$ & $\omega_{2}$ & Iter & $\omega_{1}$ & $\omega_{2}$ & Iter & & \\
\hline \multicolumn{9}{|c|}{$\alpha=1, R_{e}=10, \sigma=1.3$} \\
\hline 10 & 0.0368 & 0.0373 & 8 & 0.050 & 0.052 & 5 & $0.8036(-03)$ & $0.2692(-03)$ \\
\hline 20 & 0.0329 & 0.0334 & 9 & 0.034 & 0.046 & 6 & $0.7645(-03)$ & $0.1770(-03)$ \\
\hline 30 & 0.0297 & 0.0311 & 10 & 0.017 & 0.034 & 7 & $0.7605(-03)$ & $0.1436(-03)$ \\
\hline 40 & 0.0283 & 0.0287 & 10 & 0.023 & 0.0242 & 8 & $0.7602(-03)$ & $0.1243(-03)$ \\
\hline 50 & 0.0261 & 0.0269 & 10 & 0.025 & 0.027 & 8 & $0.7602(-03)$ & $0.1112(-03)$ \\
\hline 60 & 0.0242 & 0.0251 & 10 & 0.0211 & 0.0218 & 9 & $0.7602(-03)$ & $0.1015(-03)$ \\
\hline \multicolumn{9}{|c|}{$\alpha=2, R_{e}=10, \sigma=1.2$} \\
\hline 10 & 0.0554 & 0.0562 & 7 & 0.0555 & 0.0559 & 5 & $0.6954(-03)$ & $0.2261(-03)$ \\
\hline 20 & 0.0457 & 0.0464 & 9 & 0.035 & 0.037 & 7 & $0.4545(-03)$ & $0.1034(-04)$ \\
\hline 30 & 0.0403 & 0.0412 & 10 & 0.027 & 0.029 & 8 & $0.4245(-03)$ & $0.7896(-04)$ \\
\hline 40 & 0.0381 & 0.0393 & 10 & 0.025 & 0.030 & 9 & $0.4198(-03)$ & $0.6765(-04)$ \\
\hline 50 & 0.0359 & 0.0364 & 10 & 0.03 & 0.033 & 9 & $0.4191(-03)$ & $0.6040(-04)$ \\
\hline 60 & 0.0326 & 0.0335 & 10 & 0.022 & 0.029 & 10 & $0.4190(-03)$ & $0.5512(-04)$ \\
\hline \multicolumn{9}{|c|}{$\alpha=1, R_{e}=50, \sigma=0.9$} \\
\hline 10 & 0.0204 & 0.0211 & 8 & 0.016 & 0.020 & 7 & $0.8035(-03)$ & $0.3961(-03)$ \\
\hline 20 & 0.0183 & 0.0189 & 8 & 0.011 & 0.014 & 6 & $0.5906(-03)$ & $0.1492(-03)$ \\
\hline 30 & 0.0079 & 0.0084 & 9 & 0.007 & 0.0072 & 7 & $0.5252(-03)$ & $0.1101(-03)$ \\
\hline 40 & 0.0056 & 0.0067 & 11 & 0.0051 & 0.0052 & 9 & $0.5044(-03)$ & $0.9227(-04)$ \\
\hline 50 & 0.0036 & 0.0041 & 15 & 0.0043 & 0.0043 & 12 & $0.4973(-03)$ & $0.8159(-04)$ \\
\hline 60 & 0.0034 & 0.0035 & 17 & 0.0032 & 0.0034 & 16 & $0.4949(-03)$ & $0.7419(-04)$ \\
\hline \multicolumn{9}{|c|}{$\alpha=2, R_{e}=50, \sigma=0.9$} \\
\hline 10 & 0.0198 & 0.0211 & 8 & 0.016 & 0.017 & 7 & $0.4542(-03)$ & $0.3755(-03)$ \\
\hline 20 & 0.0172 & 0.0181 & 8 & 0.011 & 0.018 & 6 & $0.6976(-04)$ & $0.4929(-04)$ \\
\hline 30 & 0.0069 & 0.0072 & 9 & 0.007 & 0.008 & 7 & $0.4018(-04)$ & $0.2422(-04)$ \\
\hline 40 & 0.0064 & 0.0064 & 10 & 0.0065 & 0.0057 & 8 & $0.3333(-04)$ & $0.1789(-04)$ \\
\hline 50 & 0.0045 & 0.0043 & 13 & 0.0036 & 0.0041 & 12 & $0.3124(-04)$ & $0.1525(-04)$ \\
\hline 60 & 0.0035 & 0.0034 & 17 & 0.0043 & 0.0033 & 15 & $0.3054(-04)$ & $0.1372(-04)$ \\
\hline \multicolumn{9}{|c|}{$\alpha=1, R_{e}=50, \sigma=1.0$} \\
\hline 10 & 0.0174 & 0.0177 & 10 & 0.01 & 0.027 & 8 & $0.1217(-02)$ & $0.5654(-03)$ \\
\hline 20 & 0.009 & 0.011 & 12 & 0.0113 & 0.0115 & 9 & $0.1044(-03)$ & $0.4913(-04)$ \\
\hline 30 & 0.0063 & 0.0065 & 14 & 0.0072 & 0.0077 & 10 & $0.4547(-04)$ & $0.1367(-04)$ \\
\hline 40 & 0.0063 & 0.0069 & 16 & 0.0054 & 0.0056 & 11 & $0.2617(-04)$ & $0.6119(-05)$ \\
\hline 50 & 0.0042 & 0.0046 & 18 & 0.0044 & 0.0045 & 12 & $0.1697(-04)$ & $0.3416(-05)$ \\
\hline 60 & 0.0034 & 0.0039 & 21 & 0.0032 & 0.0041 & 13 & $0.1188(-04)$ & $0.2152(-05)$ \\
\hline \multicolumn{9}{|c|}{$\alpha=2, R_{e}=50, \sigma=1.0$} \\
\hline 10 & 0.017 & 0.025 & 9 & 0.015 & 0.026 & 7 & $0.1234(-02)$ & $0.5767(-03)$ \\
\hline 20 & 0.0101 & 0.011 & 11 & 0.0118 & 0.0118 & 9 & $0.1058(-03)$ & $0.4505(-04)$ \\
\hline 30 & 0.0071 & 0.0083 & 13 & 0.0075 & 0.0075 & 10 & $0.2644(-04)$ & $0.1025(-04)$ \\
\hline 40 & 0.0054 & 0.0063 & 15 & 0.0049 & 0.0058 & 11 & $0.1502(-04)$ & $0.3813(-05)$ \\
\hline 50 & 0.0043 & 0.0049 & 17 & 0.0038 & 0.0049 & 12 & $0.9686(-05)$ & $0.1870(-05)$ \\
\hline 60 & 0.0035 & 0.004 & 20 & 0.0042 & 0.0044 & 11 & $0.6762(-05)$ & $0.1084(-05)$ \\
\hline
\end{tabular}

The exact solution is given by $y(x)=x^{2} \cosh (x)$. The RMSE, MAE, and the number of iterations for both single and double sweep AGE methods are tabulated in Table 3 for various values of $\alpha, R_{e}$, and $\sigma$. The graph of Example 3 of exact solution versus numerical solution for $N=60, R_{e}=50$, $\alpha=2, \sigma=0.9$ is plotted in Figure 3 .

\section{Discussion and Conclusion}

We have discussed a new single sweep AGE iterative method and three-point off-step method of accuracy $O\left(h_{k}^{3}\right)$ on a variable mesh for the solution of nonlinear two point boundary value problems. To demonstrate the efficiency of the method, 


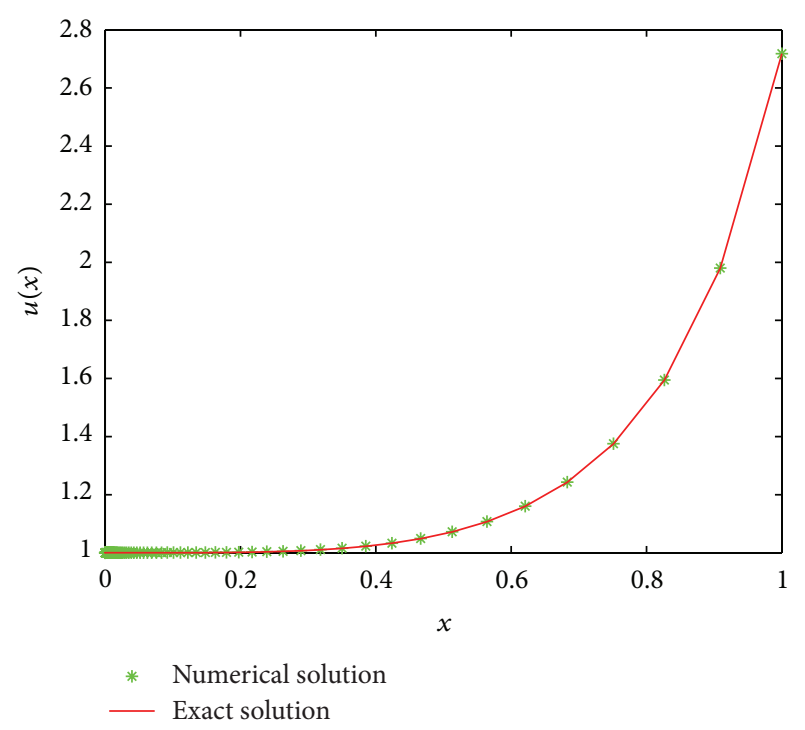

Figure 1: Graph of exact solution and the numerical solution for alpha $=2.0$, sigma $=1.1$, and $N=80$ for Example 1 .

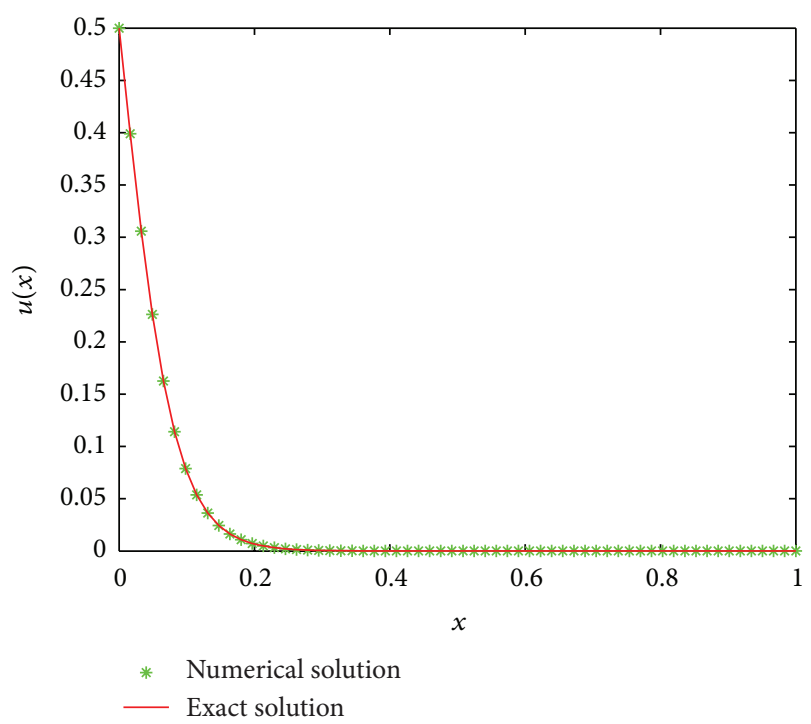

FIGURE 2: Graph of the exact solution and the numerical solution for $R=50$, sigma $=1.0$, and $N=60$ for Example 2 .

three examples including two nonlinear and singular cases are presented. The results obtained are compared with the corresponding double sweep AGE method and show superiority over the latter. The double sweep AGE method requires two sweeps to solve a problem, whereas the single sweep AGE method requires only one sweep to solve the problem. Experimentally, as compared to the double sweep method the corresponding single sweep method requires much less number of iterations as it uses less intermediate variables. The method can be extended to solve multidimensional problems and is suitable for use on parallel computers.

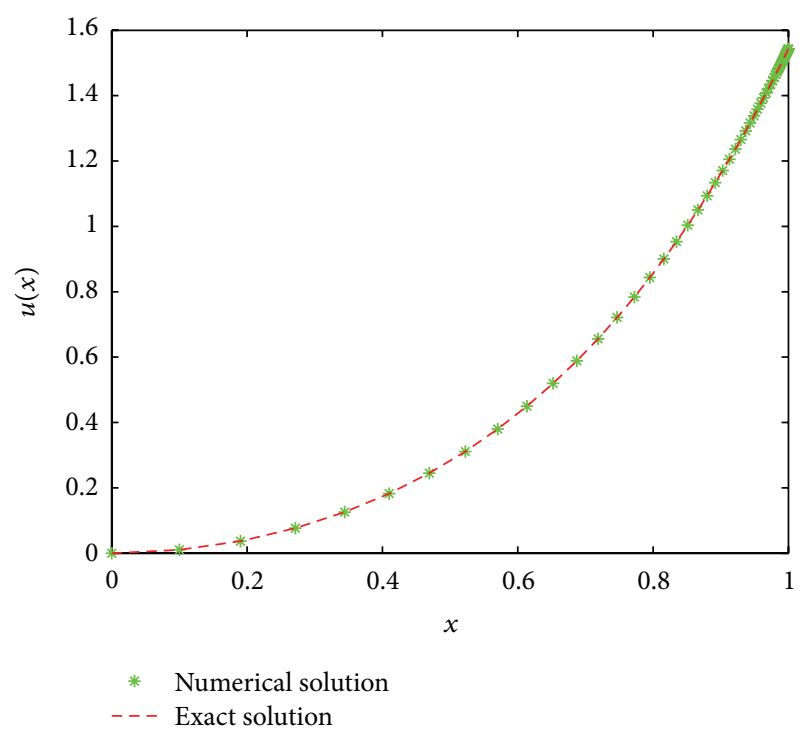

FIGURE 3: Graph of the exact solution and the numerical solution for alpha $=2.0, R=50$, sigma $=0.9$, and $N=60$ for Example 3 .

\section{Conflict of Interests}

The authors declare that there is no conflict of interests regarding the publication of this paper.

\section{Acknowledgment}

This research was supported by the Council of Scientific and Industrial Research under research Grant no. 09/045(0836) 2009-EMR-I.

\section{References}

[1] H. B. Keller, Numerical Methods for Two Point Boundary Value Problems, Blaisdell, 1968.

[2] D. J. Evans, "Parallel strategies for linear systems of equations," International Journal of Computer Mathematics, vol. 81, no. 4, pp. 417-446, 2004.

[3] D. J. Evans, "Group explicit methods for solving large linear systems," International Journal of Computer Mathematics, vol. 17, pp. 81-108, 1985.

[4] D. J. Evans and R. K. Mohanty, "Alternating group explicit method for the numerical solution of non-linear singular twopoint boundary value problems using a fourth order finite difference method," International Journal of Computer Mathematics, vol. 79, no. 10, pp. 1121-1133, 2002.

[5] R. K. Mohanty and D. J. Evans, "A fourth order accurate cubic spline alternating group explicit method for non-linear singular two point boundary value problems," International Journal of Computer Mathematics, vol. 80, no. 4, pp. 479-492, 2003.

[6] K. S. Sukon and D. J. Evans, “Two parameter AGE (TAGE) method for the solution of a tri-diagonal linear system of equations," International Journal of Computer Mathematics, vol. 60, no. 3-4, pp. 265-278, 1996.

[7] R. K. Mohanty, P. L. Sachdev, and N. Jha, "TAGE Method for nonlinear singular two point boundary value problems using a 
fourth order difference scheme," Neural, Parallel and Scientific Computations, vol. 11, pp. 281-287, 2003.

[8] D. J. Evans, "The solution of periodic parabolic equations by the coupled alternating group explicit (CAGE) iterative method," International Journal of Computer Mathematics, vol. 34, pp. 227235, 1990.

[9] D. J. Evans and Pragya Jain, "The coupled reduced alternating group explicit (CRAGE) method," Parallel Algorithms and Applications, vol. 2, pp. 193-208, 1993.

[10] Q. Feng, "Explicit finite difference method for convectiondiffusion equations," in Proceedings of the World Congress on Engineering, vol. 2, pp. 1094-1097, London, UK, 2009.

[11] B. Zheng and Q. Feng, "Parallel finite difference method for diffusion equations," in Proceedings of the 15th American Conference on Applied Mathematics, pp. 60-62, 2009.

[12] R. K. Mohanty, "A family of variable mesh methods for the estimates of (du/dr) and solution of non-linear two point boundary value problems with singularity," Journal of Computational and Applied Mathematics, vol. 182, no. 1, pp. 173-187, 2005.

[13] R. K. Mohanty, "A class of non-uniform mesh three point arithmetic average discretization for $y^{\prime \prime}=f\left(x, y, y^{\prime}\right)$ and the estimates of $y^{\prime}$," Applied Mathematics and Computation, vol. 183, no. 1, pp. 477-485, 2006.

[14] R. K. Mohanty and N. Khosla, "Application of TAGE iterative algorithms to an efficient third order arithmetic average variable mesh discretization for two-point non-linear boundary value problems," Applied Mathematics and Computation, vol. 172, no. 1, pp. 148-162, 2006.

[15] M. M. Chawla and P. N. Shivakumar, "An efficient finite difference method for two-point boundary value problems," Neural, Parallel \& Scientific Computations, vol. 4, no. 3, pp. 387-395, 1996.

[16] D. J. Evans, "Iterative methods for solving non-linear two point boundary value problems," International Journal of Computer Mathematics, vol. 72, no. 3, pp. 395-401, 1999. 

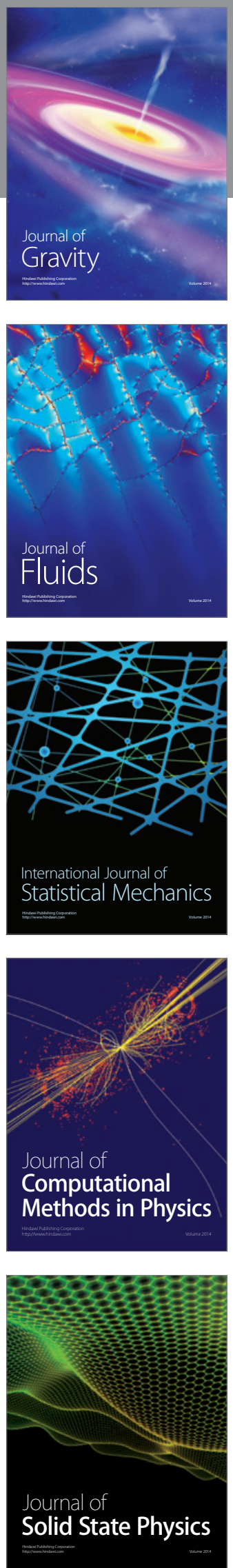

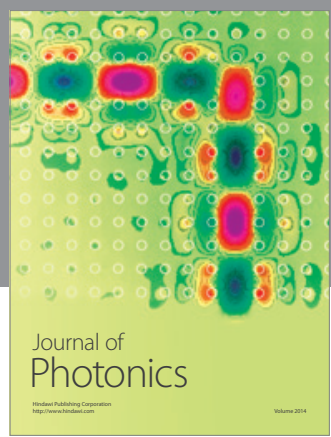

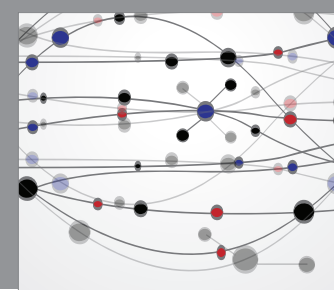

The Scientific World Journal

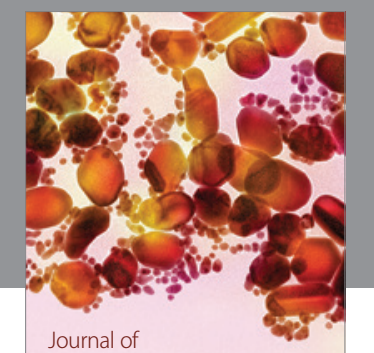

Soft Matter
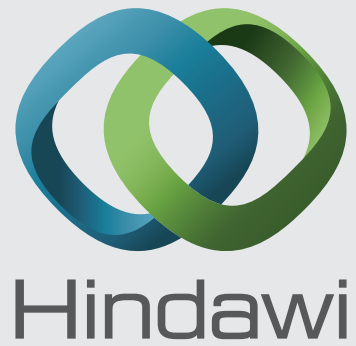

Submit your manuscripts at

http://www.hindawi.com
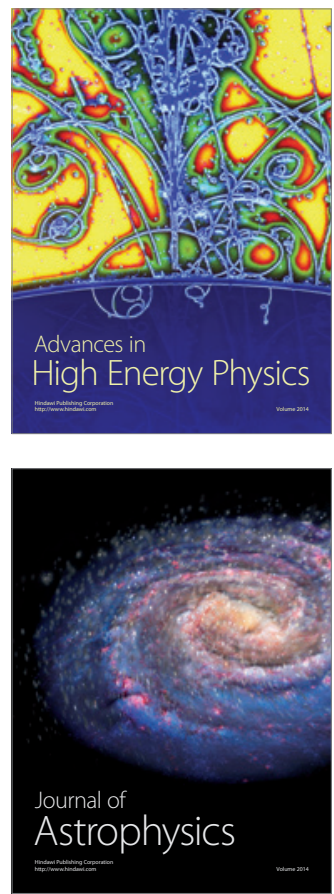
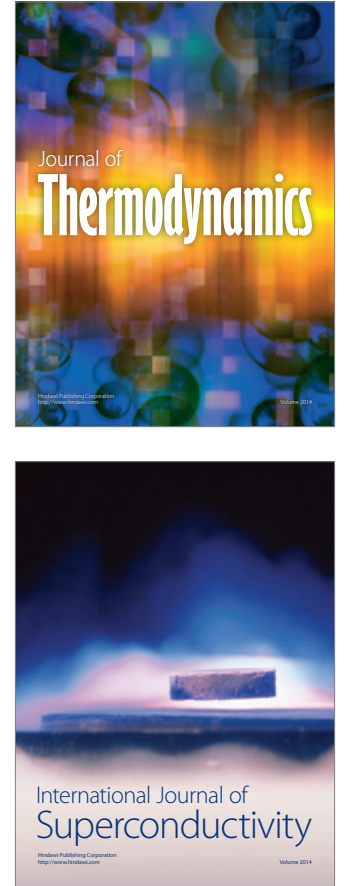
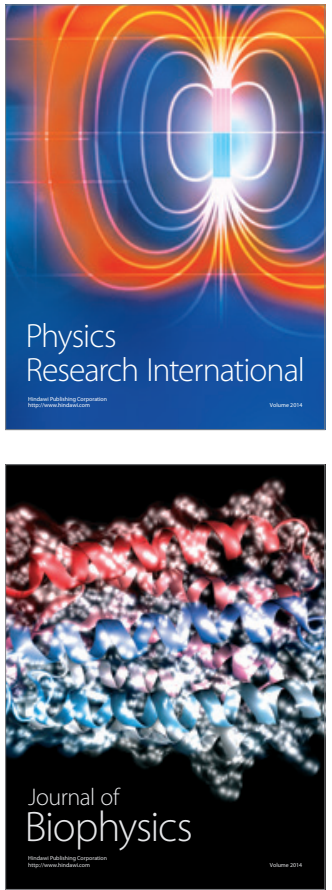
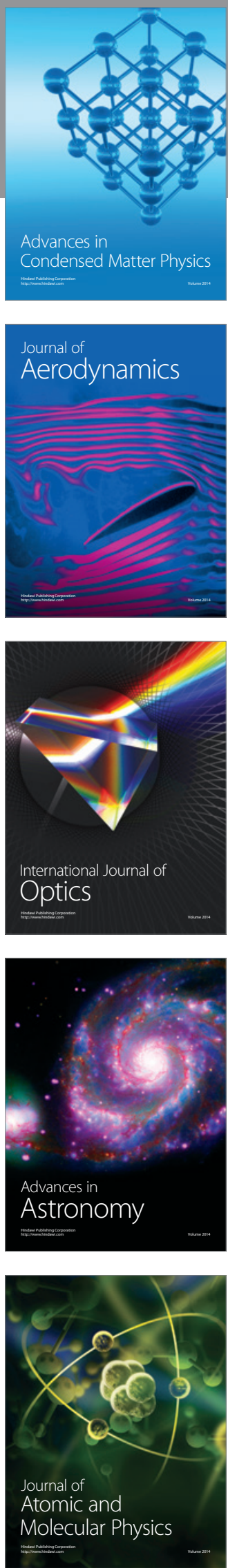